\title{
O uso da Medida Canadense de Desempenho Ocupacional em estudos brasileiros: uma revisão sistemática*
}

\author{
Using the Canadian Occupational Performance \\ Measure in Brazilian studies: a systematic review
}

\author{
Ada Salvetti Cavalcanti Caldas, Vera Lúcia Dutra Facundes², \\ Hilton Justino da Silva ${ }^{3}$
}

\begin{abstract}
CALDAS, A. S. C.; FACUNDES, V. L. D.; SILVA, H. J. O uso da Medida Canadense de Desempenho Ocupacional em estudos brasileiros: Uma revisão sistemática. Rev. Ter. Ocup. Univ. São Paulo, v. 22, n. 3, p. 238-244, set./dez. 2011.

RESUMO: O COPM caracteriza-se por ser uma medida individualizada, em que o sujeito auto-avalia as atividades mais importantes que se encontram em dificuldade em seu desempenho ocupacional. Objetivo: Revisar de forma sistemática estudos de pesquisadores brasileiros com a utilização da Medida Canadense de Desempenho Ocupacional como método avaliativo. Procedimentos metodológicos: A revisão sistemática foi realizada a partir das bases de dados PUBMED, MEDLINE (1997 - 2010), MEDLINE OLD (1966 - 1996), LILACS e SciELO. Resultados: Foram encontrados 740 artigos a partir da busca de descritores e termo livre, sendo 278 da PUBMED, 340 da MEDLINE, 56 da MEDLINE OLD, 51 da LILACS, 15 da SciELO Brasil. Considerando os critérios de inclusão e de exclusão foram selecionados 06 artigos para esta revisão. Conclusões: No Brasil há escassez de estudos com a utilização deste método avaliativo, embora internacionalmente seja um importante guia da prática clínica de terapeutas ocupacionais.
\end{abstract}

DESCRITORES: Terapia ocupacional; Avaliação de desempenho; Revisão; Brasil.

* Trabalho integrante da dissertação de mestrado em Patologia da Universidade Federal de Pernambuco - UFPE.

1. Terapeuta Ocupacional, mestranda em Patologia pela Universidade Federal de Pernambuco - UFPE; Especialista em Saúde Pública pela Universidade de Pernambuco - UPE.

2. Terapeuta Ocupacional, professora adjunta do curso de Terapia Ocupacional da Universidade Federal de Pernambuco, UFPE; Doutora em Neuropsiquiatria pela Universidade Federal de Pernambuco - UFPE; Mestre em Saúde Coletiva, PIPASC - UFPE; Especialista em Psiquiatria Social, FIOCRUZ/RJ.

3. Fonoaudiólogo, professor adjunto II da Universidade Federal de Pernambuco - UFPE; Doutor em Nutrição pela Universidade Federal de Pernambuco - UFPE; Mestre em Morfologia pela Universidade Federal de Pernambuco - UFPE; Especialista em Motricidade Orofacial, CEFAC - CFFa.

Endereço para correspondência: Ada Salvetti Cavalcanti Caldas. Rua Guedes Pereira, 180 - apto. 903 - Parnamirim, PE. CEP. 52060150. Recife, Pernambuco. E-mail: adasc@hotmail.com 


\section{INTRODUÇÃO}

$\mathrm{O}$ Desempenho Ocupacional, de acordo com a Occupacional Therapy Guidelines for Client-centred Pratice da Associação Canadense de Terapia Ocupacional, define-se como a habilidade de realizar rotinas e desempenhar papéis e tarefas, com o objetivo de autocuidado, produtividade e lazer em resposta às demandas do meio externo e interno ao indivíduo (CHAPPARO; RANKA, 1997; ZANNI et al., 2009).

Nessa perspectiva, a Medida Canadense de Desempenho Ocupacional (COPM), publicada em 1990 por Law et al., foi desenvolvida por pesquisadores canadenses com o intuito de ser utilizada como guia para terapeutas ocupacionais, baseando-se na prática centrada no cliente.

O desenvolvimento da medida teve como aspecto estruturador o Modelo Canadense de Desempenho Ocupacional, focalizando o desempenho nas áreas de autocuidado, produtividade e lazer, bem como os componentes de desempenho (físico, mental, sociocultural e espiritual), o ambiente, o estágio de desenvolvimento, os papéis na vida e a motivação do sujeito (LAW et al., 2009).

O COPM caracteriza-se por ser uma medida individualizada, realizada através de entrevista semiestruturada, em que o sujeito pontua as atividades mais importantes em seu cotidiano que se encontra em dificuldade (POLLOCK et al., 2003; LAW et al., 2009). A medida abrange três áreas de desempenho ocupacional: atividades de autocuidado (cuidados pessoais, mobilidade funcional e funcionamento na comunidade), atividades produtivas (trabalho remunerado ou não, manejo das tarefas domésticas, escola e brincar) e atividades de lazer (ação tranquila, recreação ativa e socialização). Na avaliação atribui-se um grau de importância a essas atividades, que varia numa escala de 1 a 10, de forma crescente.

O terapeuta pontua, com o cliente, os cinco principais problemas de desempenho ocupacional vivenciados, listando as atividades comprometidas conforme o grau de importância estabelecido. Em seguida, o sujeito auto-avalia seu desempenho e satisfação com esse desempenho também por meio de duas escalas de variação de 1 a 10 pontos para as respectivas tarefas funcionais.

Tendo em vista a importância do uso da COPM na avaliação funcional, bem como o desconhecimento sobre o seu uso em pesquisas brasileiras, a presente pesquisa tem como objetivo revisar, de forma sistemática, estudos de pesquisadores brasileiros com a utilização da Medida Canadense de Desempenho Ocupacional.

\section{PROCEDIMENTOS METODOLÓGICOS}

A revisão sistemática da literatura foi realizada a partir das bases de dados PUBMED, MEDLINE (19972010), MEDLINE OLD (1966-1996), LILACS e SciELO, A busca foi realizada por três pesquisadores de forma independente, seguindo os critérios de inclusão e exclusão, em janeiro de 2011.

Para a pesquisa foram utilizados descritores (DeCS) - palavras-chaves para recuperação de assuntos da literatura científica - e termos livres (TL) - termos não encontrados no DeCS e MESH, mas de relevância para a pesquisa -, sendo realizados os seguintes cruzamentos: "Occupational performance" (DeCS) AND "COPM" (TL); "Brazil" (DeCS) AND "COPM" (TL); "Employee Performance Appraisal" (DeCS) AND "COPM" (TL); "Occupational Therapy" (DeCS) AND "COPM" (TL); "Occupational Therapy" (DeCS) AND "Employee Performance Appraisal" (DeCS); "Occupational Therapy" (DeCS) AND "Brazil" (DeCS), e seus equivalentes em português e espanhol.

Como critérios de inclusão foram selecionados artigos originais que utilizaram o protocolo da Medida Canadense de Desempenho Ocupacional no Brasil, publicados nos idioma português e inglês. Os artigos de revisão da literatura, editoriais e estudo de caso foram excluídos, bem como aqueles que não propuseram pesquisar a população brasileira.

\section{RESULTADOS}

Foram encontrados 740 artigos a partir da busca de descritores e termo livre, sendo 278 da PUBMED, 340 da MEDLINE, 56 da MEDLINE OLD, 51 da LILACS, 15 da SciELO Brasil. Considerando os critérios de inclusão e de exclusão foram selecionados seis artigos para esta revisão, conforme aponta a Figura 1.

Para melhor apresentação dos resultados optouse por considerar as seguintes variáveis dos artigos selecionados: autor, ano, local, amostragem, seguimento, principais atividades comprometidas e resultados, como podem ser observados na Tabela 1. 
Tabela 1 - Estudos brasileiros que utilizaram o COPM como método avaliativo

\begin{tabular}{|c|c|c|c|c|c|}
\hline Autor/ Ano & Local & Amostragem & Seguimento & Principais atividades comprometidas & Resultados \\
\hline $\begin{array}{l}\text { Garros et } \\
\text { al., } 2010\end{array}$ & $\begin{array}{l}\text { Irmandade } \\
\text { Santa } \\
\text { Casa de } \\
\text { Misericórdia } \\
\text { de São } \\
\text { Paulo }\end{array}$ & $\begin{array}{l}30 \text { sujeitos } \\
\text { adultos com } \\
\text { espasticidade } \\
\text { em uma das } \\
\text { mãos causado } \\
\text { por AVC }\end{array}$ & $\begin{array}{l}\text { Avaliação antes } \\
\text { da intervenção e } \\
3 \text { meses após o } \\
\text { uso da órtese }\end{array}$ & $\begin{array}{l}\text { Vestuário; higiene, alimentação; } \\
\text { mobilidade; e independência fora de casa }\end{array}$ & $\begin{array}{l}\text { Obteve-se melhora em relação } \\
\text { ao desempenho após o uso de } \\
\text { órtese com média de } 1,4 \pm 0,5 \text { para } \\
6,3 \pm 0,8 \text { ( } p<0,01) \text {. Quanto à média da } \\
\text { satisfação foi de } 1,7 \pm 0,4 \text { para } 6,3 \pm 0,6 \\
(p<0,01)\end{array}$ \\
\hline $\begin{array}{l}\text { Andolfato; } \\
\text { Mariotti, } \\
2009\end{array}$ & $\begin{array}{l}\text { Clínica de } \\
\text { Doenças } \\
\text { Renais, em } \\
\text { Curitiba }\end{array}$ & $\begin{array}{l}21 \text { sujeitos } \\
\text { portadores de } \\
\text { insuficiência } \\
\text { renal crônica }\end{array}$ & $\begin{array}{l}\text { Avaliação inicial } \\
\text { e reavaliação } \\
\text { após } 16 \text { sessões } \\
\text { de Terapia } \\
\text { Ocupacional }\end{array}$ & $\begin{array}{l}\text { Controlar a dieta líquida e sólida, participar } \\
\text { de atividades sociais, freqüentar cursos, } \\
\text { praticar hobbies, realizar limpezas, realizar } \\
\text { atividades voluntárias, praticar esportes, } \\
\text { freqüentar escola, tomar remédios, } \\
\text { deambular fora de casa, utilizar ônibus, } \\
\text { lavar roupas, fazer artesanato, realizar } \\
\text { visitas, viajar, deambular dentro de casa, } \\
\text { retornar ao trabalho, banhar-se, fazer } \\
\text { compras, cortar grama, manter-se no } \\
\text { emprego, cortar alimentos (preparo de } \\
\text { refeições), receber auxílio doença, realizar } \\
\text { passeios. }\end{array}$ & $\begin{array}{l}\text { Os maiores problemas de } \\
\text { desempenho ocupacional foram } \\
\text { controlar a dieta líquida e sólida, } \\
\text { participar de atividades sociais e } \\
\text { freqüentar cursos. O percentual de } \\
\text { sujeitos que apresentou variação } \\
\text { de } 2 \text { ou mais pontos nos escores } \\
\text { de satisfação e desempenho foi de } \\
52,4 \% \text { e } 47,6 \% \text {, respectivamente. }\end{array}$ \\
\hline $\begin{array}{l}\text { Nickel et } \\
\text { al., } 2009\end{array}$ & $\begin{array}{l}\text { Instituição } \\
\text { para DP em } \\
\text { Curitiba }\end{array}$ & $\begin{array}{l}46 \text { sujeitos } \\
\text { diagnosticados } \\
\text { com DP }\end{array}$ & Avaliação única & $\begin{array}{l}\text { De acordo com a CIF: Vida comunitária, } \\
\text { social e cívica; mobilidade; cuidado } \\
\text { pessoal; vida doméstica e aprendizagem; } \\
\text { e aplicação de conhecimento }\end{array}$ & $\begin{array}{l}\text { O modelo proposto pela CIF em } \\
\text { conjunto com a aplicação da COPM } \\
\text { mostrou-se efetivo, permitindo } \\
\text { correlação quando a atividade é } \\
\text { foco de avaliação, entre funções } \\
\text { e estruturas do corpo, fatores } \\
\text { ambientais e pessoais, com as } \\
\text { dificuldades de desempenho na } \\
\text { realização das atividades. }\end{array}$ \\
\hline $\begin{array}{l}\text { Zanni et } \\
\text { al., } 2009\end{array}$ & $\begin{array}{l}\text { FAMERP - } \\
\text { São José do } \\
\text { Rio Preto }\end{array}$ & $\begin{array}{l}30 \text { pacientes } \\
\text { epilépticos } \\
\text { adultos }\end{array}$ & $\begin{array}{l}\text { Aplicação } 1 \\
\text { mês antes do } \\
\text { tratamento } \\
\text { cirúrgico e } \\
\text { reaplicação } 12 \\
\text { meses após a } \\
\text { intervenção }\end{array}$ & $\begin{array}{l}\text { Conseguir um emprego/trabalhar; lembrar- } \\
\text { se de nomes, endereços, telefones ou } \\
\text { do local onde foram guardados objetos } \\
\text { pessoais; cozinhar; utilizar transporte } \\
\text { público; utilizar dinheiro ou administrar } \\
\text { contas e despesas da casa; sair sozinho } \\
\text { de casa; lavar roupas; dirigir e frequentar } \\
\text { escola; ir a festa ou passeio; namorar/ } \\
\text { ter um relacionamento; conversar com } \\
\text { amigos; visitar parentes; viajar com a } \\
\text { família; andar de bicicleta e ir à igreja. }\end{array}$ & $\begin{array}{l}\text { A comparação dos resultados do } \\
\text { COPM do pré- com o pós-operatório } \\
\text { mostrou um aumento significativo } \\
\text { tanto na performance quanto na } \\
\text { satisfação dos pacientes com suas } \\
\text { AVD, AIVD e lazer. }\end{array}$ \\
\hline $\begin{array}{l}\text { Figueiredo } \\
\text { et al., } 2006\end{array}$ & $\begin{array}{l}\text { Hospital } \\
\text { Público } \\
\text { de Belo } \\
\text { Horizonte }\end{array}$ & $\begin{array}{l}42 \text { pacientes } \\
\text { com lesão } \\
\text { traumática } \\
\text { da mão em } \\
\text { decorrência } \\
\text { de acidente de } \\
\text { trabalho }\end{array}$ & $\begin{array}{l}\text { Avaliação } \\
\text { administrada em } \\
\text { três momentos: } \\
\text { na admissão, na } \\
\text { alta e follow-up } \\
\text { de um mês }\end{array}$ & $\begin{array}{l}\text { Amarrar calçados; tomar banho; escovar } \\
\text { dentes; cortar unha; abotoar roupas; fazer } \\
\text { barba; servir prato; varrer casa; lavar } \\
\text { roupa; preparar refeições; lavar louça ou } \\
\text { vasilha; picar verdura e legumes; carregar } \\
\text { sacola; segurar-se no ônibus; firmar } \\
\text { ferramentas de trabalho e peças pequenas } \\
\text { e carregar peso; }\end{array}$ & $\begin{array}{l}\text { Os escores do COPM atestaram que } \\
\text { houve melhora significativa entre a } \\
\text { admissão e a alta e também entre a } \\
\text { admissão e o follow-up. Porém não foi } \\
\text { constatada diferença estatisticamente } \\
\text { significativa entre as avaliações } \\
\text { realizadas no momento da alta e do } \\
\text { follow-up. }\end{array}$ \\
\hline $\begin{array}{l}\text { Sampaio et } \\
\text { al., } 2006\end{array}$ & $\begin{array}{l}\text { Hospital } \\
\text { Público } \\
\text { de Belo } \\
\text { Horizonte }\end{array}$ & $\begin{array}{l}42 \text { pacientes } \\
\text { com lesão } \\
\text { traumática } \\
\text { da mão em } \\
\text { decorrência } \\
\text { de acidente de } \\
\text { trabalho }\end{array}$ & $\begin{array}{l}\text { Avaliação } \\
\text { administrada em } \\
\text { dois momentos: } \\
\text { admissão e alta }\end{array}$ & $\begin{array}{l}\text { Tomar banho; varrer o chão; fazer o prato; } \\
\text { lavar roupa; segurar-se no ônibus; abotoar } \\
\text { roupas; amarrar calçados; carregar } \\
\text { sacolas; servir pratos; preparar refeições; } \\
\text { barbear-se; pica legumes; segurar } \\
\text { ferramentas e objetos pequenos; escovar } \\
\text { os dentes; cortar unhas e carregar peso; }\end{array}$ & $\begin{array}{l}\text { Observou-se melhora significante } \\
\text { em todos os componentes funcionais } \\
\text { avaliados. Os valores do COPM } \\
\text { aumentaram mais de } 100 \% \text { após a } \\
\text { intervenção, demonstrando melhoria } \\
\text { tanto no desempenho quanto na } \\
\text { satisfação dos clientes. }\end{array}$ \\
\hline
\end{tabular}

AVC: Acidente Vascular Cerebral; DP: Doença de Parkinson; COPM: Medida Canadense de Desempenho Ocupacional; CIF: Classificação Internacional de Funcionalidade; AVD: Atividades de Vida Diária; AIVD: Atividades Instrumentais de Vida Diária. 


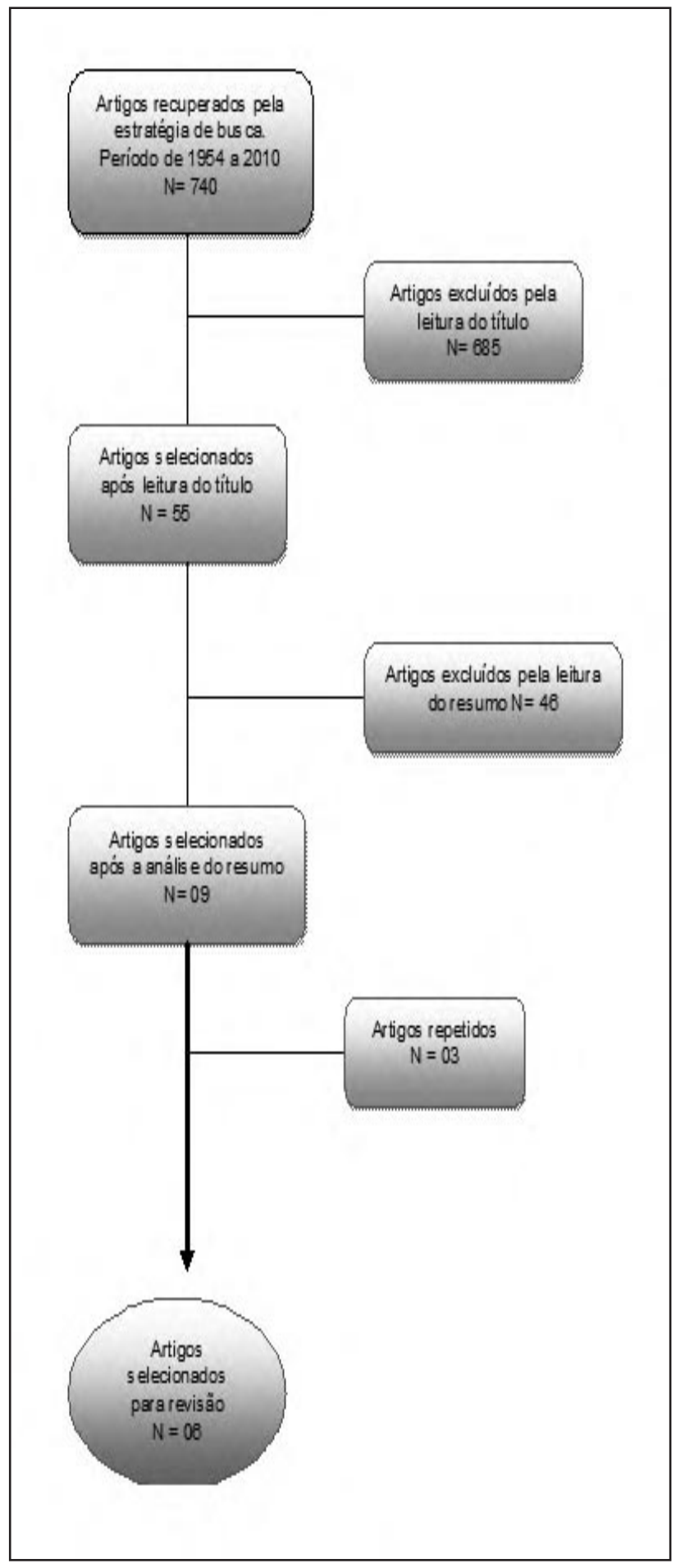

Figura 1 - Fluxograma do número de artigos encontrados e selecionados após aplicação dos critérios de inclusão e exclusão segundo descritores e bases de dados

\section{DISCUSSÃO}

A grande diversidade observada nos estudos encontrados não permitiu análise estatística (metanálise). A heterogeneidade pôde ser percebida com relação à ausência de critérios de randomização e diversificação das variáveis consideradas na em cada artigo analisado.

No Brasil, o uso da Medida Canadense de Desempenho Ocupacional (COPM) iniciou-se no ano de 2006 e embora a primeira versão para o português tenha ocorrido no ano de 1999 (MAGALHÃES et al., 2009), esta não chegou a ser publicada, o que justifica o uso escasso desse instrumento na literatura brasileira.

No ano de 2009 foi publicada, na região sudeste do Brasil, a primeira edição em língua portuguesa do manual de aplicação do COPM, supondo-se que, em decorrência desse pioneirismo, há uma concentração de estudos com a utilização deste protocolo nessa região do país.

As publicações enfocaram principalmente os componentes motores em sujeitos adultos (FIGUEIREDO et al., 2006; SAMPAIO et al., 2006; GARROS et al., 2010), embora tenha sido encontrada certa heterogeneidade nas patologias abordadas e no número de sujeitos pesquisados.

Estudos referem que o COPM tem grande impacto na prática clínica e que pode ser útil para clientes com diferentes incapacidades, independente de diagnósticos específicos (McCOLL et al., 2005; PARKER; SYKES, 2006).

Carswell et al. (2009), em uma revisão da literatura, referiram algumas limitações na utilização do COPM, não sendo um instrumento aplicável a todos os clientes, embora, de modo geral, seja uma medida com desfechos confiáveis, válidos, úteis, clinicamente aceitáveis e receptivo para os profissionais da Terapia Ocupacional e pesquisadores.

A literatura internacional tem demonstrado o uso do COPM com outras clientelas, abordando intervenções com crianças e a percepção dos pais com relação ao desempenho destas, alterando a perspectiva de quem de fato é o cliente (DONOVAN et al., 2005; KAISER et al., 2005; CUSICK et al., 2007; PHELAN et al., 2009).

Considerando-se os diversos contextos de aplicação do COPM, este instrumento pode ser utilizado com as pessoas que fazem parte do ambiente do sujeito, como a família, cuidadores ou professores. No caso do cliente ter dificuldade de comunicação, um intermediário poderá auxiliar na expressão das intenções e das opiniões desse sujeito a ser avaliado (HOBSON, 2003; OLIVEIRA; CASTANHARO, 2008; LAW et al., 2009).

Outros estudos analisaram a clientela com transtornos 
psiquiátricos, evidenciando que, embora seja um desafio, o COPM pode ser aplicado satisfatoriamente, sendo importante analisar as diferenças entre os julgamentos dos clientes e o desempenho real, bem como avaliar a viabilidade da Prática Clínica Centrada no Cliente (PAN et al., 2003; SCHINDLER, 2010). Entende-se que embora possa haver divergências entre o que é percebido pelo terapeuta e o que é exposto pelo cliente, a abordagem é realizada baseada na perspectiva do que é significativo para o sujeito.

Nesta revisão, observaram-se equívocos com relação ao método de aplicação do COPM em estudo realizado com 30 sujeitos epilépticos (ZANNI et al., 2009), utilizando valores incorretos nas escalas de desempenho e de satisfação, além de ter sido constatada nesta mesma sessão, referências não correspondentes ao estudo desse instrumento.

Com relação ao seguimento das publicações abordadas nesta revisão observou-se que no artigo que estudou sujeitos com doença de Parkinson (NICKEL et al., 2010) não houve a proposta de atestar a eficácia de um método de intervenção, sendo apenas caracterizada a população estudada com a utilização conjunta dos domínios da Classificação Internacional de Funcionalidade (CIF). Neste caso, não foi aferido o desempenho nem a satisfação dos clientes com esse desempenho, sendo pontuadas apenas as principais dificuldades.

A pontuação do desempenho e da satisfação do cliente devem ser medidas com a proposta de se reavaliar e comparar esses domínios para, assim, comprovar a eficácia do tratamento, já que a confiabilidade para a utilização do COPM está relacionada ao teste e reteste (LAW et al., 2009). No estudo de Nickel et al. (2010), a pontuação dos principais problemas de desempenho teve a proposta de caracterizar uma população, já que não foi realizada nenhum tipo de intervenção.

Outro ponto relevante foi a ocorrência da publicação de dois artigos no ano de 2006, por um grupo de pesquisadores de Belo Horizonte, com a mesma população de estudo e método investigativo semelhante (FIGUEIREDO et al., 2006; SAMPAIO et al., 2006), divergindo apenas quanto ao seguimento da pesquisa, tendo em vista que em uma das publicações foi proposta reavaliação após 1 mês de followup (FIGUEIREDO et al., 2006).

No que se refere às principais atividades comprometidas listadas nos artigos desta revisão, observou-se que grande parte das atividades abordou as áreas de autocuidado e de produtividade em detrimento das atividades lazer. Entende-se que o prejuízo motor mais comumente relatado na população dos estudos selecionados, principalmente no que se refere às lesões de mão (GARROS et al., 2010; FIGUEIREDO et al., 2006; SAMPAIO et al., 2006), podem ter determinado maior comprometimento na realização das atividades mais básicas que exigem movimentos refinados das mãos.

Em contrapartida, no estudo realizado com 21 sujeitos com Insuficiência Renal Crônica (ANDOLFATO; MARIOTTI, 2009), observou-se que atividades diversas foram relatadas como prejudicadas, e embora tenham sido majoritariamente listados os prejuízos motores no que se refere à alimentação, também foram apontados como prioritários os problemas referentes à participação social, com limitações nas três áreas do desempenho ocupacional.

Outro fator que deve ser levado em conta é a diversidade de contextos culturais e de condições de vida distintas de cada indivíduo, que podem interferir na determinação e percepção de prioridades, tendo em vista metas relevantes para o cliente, refletindo o desejo desse indivíduo no direcionamento de seu tratamento.

Molinier (2003) questiona o conceito de sujeito e relata que o ser humano não é definido por condições e contextos externos, mas por características internas. Ao ampliar o conceito de subjetividade, Galheigo (2003) pontua os significados que as pessoas dão às suas experiências, na compreensão e interpretação da sua realidade, indo de encontro aos seus desejos e anseios, defendendo ainda que o cotidiano traz em si a marca da singularidade do sujeito, modelando-se a partir de suas necessidades, valores, crenças e afetos.

Os resultados apontados nesta revisão corroboraram a utilidade deste protocolo descrita anteriormente por McColl et al. (2000), demonstrando que o COPM pode ser um instrumento válido para atestar a eficácia de uma intervenção, partindo da perspectiva do cliente quanto a auto-avaliação de seu desempenho e satisfação.

Garros et al. (2009) em estudo realizado com 30 pacientes com mão espástica, comprovaram que após utilização de órtese dorsal volar houve melhora efetiva no desempenho ocupacional e satisfação desses sujeitos, tendo o COPM sido um instrumento relevante na análise da avaliação funcional.

\section{CONCLUSÕES}

O COPM é um instrumento válido com ampla aplicabilidade, embora, em alguns casos, necessite de adaptações ou reestruturação da forma de pensar no cliente. Como protocolo Centrado na Prática Baseada no Cliente é possível absorver as reais concepções do sujeito sobre o desempenho em atividades as quais ele julga prejudicadas, bem como a satisfação com o mesmo, trabalhando com 
perspectivas e metas significantes para o indivíduo.

No Brasil, há escassez de estudos com a utilização deste método avaliativo, embora internacionalmente seja um importante guia da prática clínica de terapeutas ocupacionais. Espera-se que novos estudos sejam estimulados com a utilização desse protocolo na prática clínica e na produção científica brasileira, facilitando a abordagem centrada no cliente.

CALDAS, A. S. C.; FACUNDES, V. L. D.; SILVA, H. J. Using the Canadian Occupational Performance Measure in Brazilian studies: a systematic review. Rev. Ter. Ocup. Univ. São Paulo, v. 22, n. 3, p. 238-244, set./dez. 2011.

\begin{abstract}
The COPM is characterized by a measure to be individualized, in which the subject self-assessment the activities most important that are in difficulty in their occupational performance. Objective: Systematically review studies of Brazilian researchers using the Canadian Occupational Performance Measure as evaluation method. Methodological procedures: A systematic review was performed from the PUBMED, MEDLINE (1997 - 2010), MEDLINE OLD (1966 - 1996), LILACS and SciELO. Results: There were 740 articles from the search term descriptors and free, and 278 of the PUBMED, 340 of the MEDLINE, 56 of the MEDLINE OLD, 51 of the LILACS, 15 of the SCIELO Brazil. Considering the criteria for inclusion and exclusion of 06 articles were selected for this review. Conclusions: In Brazil there are few studies using this evaluation method, although internationally is an important guide for clinical practice of occupational therapists.
\end{abstract}

KEY WORDS: Occupational therapy; Employee performance appraisal; Review; Brazil.

\title{
REFERÊNCIAS
}

ANDOLFATO, C.; MARIOTTI, M. C. Avaliação do paciente em hemodiálise por meio da medida canadense de desempenho ocupacional. Rev. Ter. Ocup. Univ. São Paulo, v. 20, n. 1, p. 1-7, 2009.

CARSWELL, A.; McCOLL, M. A.; BAPTISTE, S.; LAW, M.; POLATAJKO, H.; POLLOCK, N. The Canadian Occupational Performance Measure: a research and clinical literature review. Can. J. Occup. Ther.,v. 71, n. 4, p. 210-222, 2004.

CHAPPARO, C.; RANKA, J. The Occupational Performance Model (Australia): A description of constructs and structure. Sidney, Australia: The University of Sidney, 1997. p. 1-22.

CUSICK, A.; LANNIN, N. A.; LOWE, K. Adapting the Canadian Occupational Performance Measure for use in a paediatric clinical trial. Disabil. Rehabil., v. 29, n. 10, p. 761-766, 2007.

DONOVAN, J. M.; VANLEIT, B. J.; CROWE, T. K.; KEEFE, E. B. Occupational goals of mothers of children with disabilities: influence of temporal, social, and emotional contexts. Am. J. Occup. Ther., v. 59, n. 3, p. 249-261, 2005.

FIGUEIREDO, I. M.; SAMPAIO, R. F.; MANCINI, M. C.; NASCIMENTO, M. C. Ganhos funcionais e sua relação com os componentes de função em trabalhadores com lesão de mão. Rev. Bras. Fisioter, v. 10, n. 4, p. 421-427, 2006.
GALHEIGO, S. M. O cotidiano na terapia ocupacional: cultura, subjetividade e contexto históricosocial. Rev. Ter. Ocup. Univ. São Paulo, v. 14, n. 3, p. 104-109, 2003.

GARROS, D. S. C.; GAGLIARDI, R. J.; GUZZO, R. A. R. Evaluation of performance and personal satisfaction of the patient with spastic hand after using a volar dorsal orthosis. Arq. Neuropsiquiatr., v. 68, n.3, p. 385-389, 2010.

HOBSON, S. J. G. Emprego da abordagem baseada no cliente em pessoas com alterações cognitivas. In: SUMSION, T. Prática baseada no cliente na terapia ocupacional: guia para implementação. São Paulo: Roca, 2003. p. 89-107.

KAISER, M. L.; BRAUN, M.; RHYNER, C. Utilization of the Canadian Occupational Performance Measure (COPM) among children and their parents: a Swiss experience. Can. J. Occup. Ther., v. 72, n. 1, p. 30-36, 2005.

LAW, M.; BAPTISTE, S.; McCOLL, M. A.; OPZOOMER, A.; POLATAJKO, H.; POLLOCK. N. The Canadian Occupational Performance Measure: An Outcome Measure for Occupational Therapy. Can. J. Occup. Ther., v. 57, n. 2, p. 82-87, 1990.

LAW, M.; BAPTISTE, S.; CARSWELL, A.; McCOLL, M. A.; POLATAJKO, H. L.; POLLOCK, N. Medida Canadense de Desempenho Ocupacional (COPM). Trad. Lívia de Castro 
Magalhães, Lilian Vieira Magalhães e Ana Amélia Cardoso. Belo Horizonte: Editora Universidade Federal de Minas Gerais, 2009.

MAGALHÃES, L. C.; MAGALHÃES, L. V.; CARDOSO, A. A. Apresentação. In: LAW, M.; BAPTISTE, S.; CARSWELL, A.; McCOLL, M. A.; POLATAJKO, H.; POLLOCK, N. Medida Canadense de Desempenho Ocupacional (COPM). Tradução e organização de Lívia de Castro Magalhães, Lilian Vieira Magalhães e Ana Amélia Cardoso. Belo Horizonte: Editora Universidade Federal de Minas Gerais, 2009. p. 11.

McCOLL, M. A.; LAW, M.; BAPTISTE, S.; POLLOCK, N.; CARSWELL, A.; POLATAJKO, H. J. Targeted applications of the Canadian Occupational Performance Measure. Can. J. Occup. Ther., v. 72, n. 5, p. 298-300, 2005.

McCOLL, M. A.; PATERSON, M.; DAVIES, D.; DOUBT, L.; LAW, M. Validity and community utility of the Canadian Occupational Performance Measure Canadian. Can. J. Occup. Ther., v. 67, p. 22-30, 2000.

MOLINIER, P. Sujeito e subjetividade: questões metodológicas em psicodinâmica do trabalho. Rev. Ter. Ocup. Univ. São Paulo, v. 14, n. 1, p. $43-47,2003$.

NICKEL, R.; PINTO, L. M.; LIMA, A. P.; NAVARRO, E. J.; TEIVE, H. A. G.; BECKER, N.; MUNHOZ, R. P. Estudo descritivo do desempenho ocupacional do sujeito com doença de Parkinson: o uso da CIF como ferramenta para classificação da atividade e participação. Acta Fisiatr., v. 17, n. 1, p. 13-17, 2010.

OLIVEIRA, C.; CASTANHARO, R. C. T. O terapeuta ocupacional como facilitador do processo educacional de crianças com dificuldades de aprendizagem. Cad. Ter. Ocup. UFSCar, v. 16, n. 2, p. 91-99, 2008.

PAN, A. W.; CHUNG, L.; HSIN-HWEI, G. Reliability and validity of the Canadian Occupational Performance Measure for clients with psychiatric disorders in Taiwan. Occup. Ther. Int., v. 10, n. 4, p. 269-277, 2003.

PARKER, D.; SYKES, C. H. A systematic review of the Canadian Occupational Performance Measure: a clinical practice perspective. Britsh J. OccupTher., v. 69, n. 4, p. 150-160, 2006.

PHELAN, S.; STEINKE, L.; MANDICH, A. Exploring a cognitive intervention for children with pervasive developmental disorder. Can. J. Occup. Ther, v. 76, n. 1, p. 23-28, 2009.

POLLOCK, N.; McCOLL, M. A.; CARSWELL, A. Medida de Performance Ocupacional Canadense. In: SUNSION, T. (Ed.). Prática baseada no cliente na terapia ocupacional: guia para implementação. São Paulo: Roca, 2003. p. 183-204.

SAMPAIO, R. F.; MANCINI, M. C.; SILVA, F. C. M.; FIGUEIREDO, I. M.; VAZ, D. V.; ALVES, G. B. O. Work-related hand injuries: case analyses in a Brazilian rehabilitation service. Disabil. Rehabil., v. 28, n. 12, p. 803-808, 2006.

SCHINDLER, V.P.A client-centred, occupation-based occupational therapy programme for adults with psychiatric diagnoses. Occup. Ther. Int., v. 17, n. 3, p. 105-112, 2010.

ZANNI, K. P.; BIANCHIN, M. A.; MARQUES, L. H. N. Qualidade de vida e desempenho ocupacional de pacientes submetidos à cirurgia de epilepsia. J. Epilepsy Clin. Neurophysiol., Porto Alegre, v. 15, n. 3, p. 114-117, 2009 